\title{
Spirituality and Schizophrenia - Case Report and Literature Review
}

\author{
Fátima Urzal ${ }^{1}$, Inês Donas-Boto1, Sónia Farinha Silva², Rui Martins ${ }^{1}$ \\ 1 - Department of Psychiatry and Mental Health, Centro Hospitalar de Lisboa Ocidental \\ 2 - Department of Psychiatry, Unidade de Saúde Local do Baixo Alentejo
}

\section{Objectives}

To characterize the complex relationships between schizophrenia, spirituality and religion.

\section{Background and aims}

Spirituality influences the presentation, course and outcome of schizophrenia. The authors report an illustrative clinical case and conduct a literature review on this subject.

\section{Materials and methods}

Pubmed database was searched from 2004 to 2019 using the terms "schizophrenia" and "religion". Relevant data from the patient's medical record was collected.

\section{Results and Conclusions}

Pesented with extensive self-reference and religious delusions and attenuation of the boundaries of self, namely passivity
phenomena - the involuntary movement of his members into a lotus position. He interpreted this last phenomenon as a
spiritual initiation in Rosicrucianism, organization of which he had been part for several years.
He rejected the schizophrenia diagnosis and presented poor treatment adherence.

The prevalence of religious themes in psychosis varies between cultures and across time, highlighting the relevance of cultural context. ${ }^{1}$

Overextended theory of mind and agency overdetection are core cognitive concepts in the genesis of both religious experience and productive symptoms. ${ }^{3}$ Delusions of reference may be similar to the finding of religious meaning and purpose in mundane experiences and religious experience involves, to some degree, the alteration in the sense of agency, in an obvious parallel with passivity phenomena. ${ }^{3}$

While there is emerging evidence for a continuum between religious normality and psychosis ${ }^{1,2}$ and phenomenological parallels can be drawn, ${ }^{3}$ the hallmarks of pathological states include:

\section{Subjective experience and behaviour are recognizable as part of a known psychiatric illness and/or other symptoms are present ${ }^{4}$}

\section{The behaviour and direction of personal goals after the religious experience are more consistent with mental illness than with an enriching life experience ${ }^{4}$}

There is a previous long-lasting sense of being ontologically different ${ }^{5}$

The mystical experiences are trait-like (versus episodic) and private 5 ; and can't seem to be inteintegrated in reality afterwards ${ }^{5} \rightarrow$ double book keeping 6

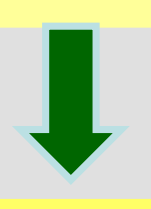

Religious themes are

Held with greater conviction than other types of delusions ${ }^{7}$

Associated with more severe illness ${ }^{7}$ and poorer functioning ${ }^{9}$

Positive effects of religion on quality of life seem to be mediated through intrinsic religion (religion as a framework to understand life), while the global impact of extrinsic religion is unknown. ${ }^{10}$

Accordingly, although a consistent belief system is associated with positive coping skills, ${ }^{11,12}$ results on the influence of religious experience on quality of life and disease outcomes have been mixed. 9,12,13,14

Further research is needed, namely regarding treatment adherence. 